\title{
White-light toxicity, resulting from systemically administered 5-aminolevulinic acid, under normal operating conditions
}

\author{
Maurice C.G. Aalders ${ }^{a}$, Nine v.d. Vange ${ }^{\text {b, }}$, Fiona A. Stewart ${ }^{c}$, Marloes G. Klein ${ }^{\mathrm{d}}$, \\ Mark J. v.d. Vijver ${ }^{\mathrm{e}}$, Henricus J.C. M. Sterenborg ${ }^{\mathrm{f}}$ \\ ${ }^{a}$ Laser Centre, Academic Medical Centre, Amsterdam, The Netherlands \\ ${ }^{\mathrm{b}}$ Department of Gynecology, Antoni van Leeuwenhoek Hospital, Amsterdam, The Netherlands \\ ${ }^{\mathrm{c}}$ Division of Experimental Therapy, Antoni van Leeuwenhoek Hospital, Amsterdam, The Netherlands \\ ${ }^{d}$ Department of Experimental Surgery, Academic Medical Centre, Amsterdam, The Netherlands \\ ${ }^{\mathrm{e}}$ Department of Pathology, Antoni van Leeuwenhoek Hospital, Amsterdam, The Netherlands \\ ' Department of Radiotherapy, Daniel den Hoed Cancer Centre, Rotterdam, The Netherlands
}

Received 25 May 1999; accepted 3 June 1999

\begin{abstract}
This study has investigated damage to the intraperitoneal organs of the rat after systemic (intraperitoneal and intravenous) administration of low doses of 5-aminolevulinic acid (ALA) and illumination with a standard white-light operating-room (o.r.) lamp. The study has been done within the framework of a larger study in which the possibility of using ALA for localization of small-volume macroscopically nonvisible peritoneal metastasis of ovarian tumors is being investigated. Fluorescence diagnostics are done in addition to the standard staging and localization procedures, either through a laparoscope or during laparotomy. In these circumstances, fluorescence diagnostics involve some risk of photosensitization of critical organs since a broad-band (o.r.) light source is used during the surgical procedures for illumination of the operating area. The drug dose and the time interval between administration of ALA and illumination are varied and normal tissues are examined both macroscopically and microscopically for damage. A relationship is demonstrated between the maximum tolerable dose (MTD) of ALA (defined as the dose that does not cause any tissue damage) and the time interval between administration and illumination. The white light that is used for illumination of the operating area is sufficient to induce damage to the peritoneal organs at relatively low ALA doses. The MDTs for 2, 6 and $16 \mathrm{~h}$ intervals are found to be respectively 1,10 and $100 \mathrm{mg} \mathrm{kg}^{-1}$. The results are similar for both intraperitoneal and intravenous administration.

(C) 1999 Elsevier Science S.A. All rights reserved.
\end{abstract}

Keywords: 5-Aminolevulinic acid; Fluorescence; White-light toxicity; Tissue damage; Abdominal cavity

\section{Introduction}

In the majority of patients with ovarian cancer the diagnosis is established at an advanced stage, when spreading into the abdominal cavity has already occurred. The standard staging procedure in ovarian cancer without overt spread is done by taking multiple blind biopsies at random from peritoneal surfaces for microscopical examination. The risk of understaging and subsequent undertreatment with this non-directed staging procedure is high.

The problem of possible understaging may be solved by using a fluorescence diagnostic technique as a tool for tumor localization. In this technique, a fluorescent dye is administered and distributed in the body, resulting in concentration gradients which can be used to discriminate normal from

\footnotetext{
* Corresponding author. Fax: + 31-20-512-2554; E-mail: vdvange@nki.nl
}

tumor tissue. 5-Aminolevulinic acid (ALA)-induced protoporphyrin IX ( $\mathrm{PpIX}$ ) is an effective fluorescent agent, which has been successfully used for the detection and localization of various malignancies [1-6]. Hornung et al. [1] showed that in ovarian cancer ALA may improve detection of superficial malignant lesions in the abdominal cavity during staging and second-look surgery by enabling directed non-blind biopsies to be taken.

ALA is metabolized to PpIX, a precursor of heme, which will selectively accumulate in tumor tissue. Apart from being fluorescent, $\mathrm{PpIX}$ is also a potent photosensitizer [6-21]. For diagnostic applications the photosensitizing effect is unwanted, as it may cause damage to healthy tissue. The extent of photosensitization depends on the dosage of ALA and the interval between administration of ALA and exposure to light, and is highly dependent on the properties of the 
excitation light source. Fluorescence diagnostics in ovarian cancer are carried out during staging laparotomy or laparoscopy. Photosensitized tissue will then be exposed to broad white light from the operating-room (o.r.) lamp or endoscopic light source. Concern arises as to whether ALA will induce damage to critical abdominal organs when they are exposed to white light. Hornung et al. [1] did not report on this aspect.

In the present study we have investigated the macroscopic and microscopic damage caused by ALA-induced PpIX to the abdominal organs of the rat when exposed during laparotomy to a white-light o.r. lamp. Several ALA doses and time intervals between ALA administration and light exposure have been tested and intraperitoneal (i.p.) administration of ALA is compared to intravenous (i.v.) administration. The aim of our study is to establish a range of doses of ALA in combination with different time intervals that can be used safely in staging procedures for ovarian cancer without the appearance of harmful photosensitization effects to abdominal tissues.

\section{Materials and methods}

\subsection{Animals, drug dose and illumination properties}

Adult female Wistar rats (i.p. injections; $n=41$ ) or Wag/ Rij rats (i.v. injections; $n=22$ ) of approximately $200 \mathrm{~g}$ body weight were used. The animals received ALA dissolved in PBS by an i.p. or i.v. injection. The dose of i.p. ALA varied from 1 to $200 \mathrm{mg} \mathrm{kg}^{-1}$ and the time interval between ALA and illumination during laparotomy was $2,6,16$ or $24 \mathrm{~h}$. For the i.v. administration route, drug doses of $1-100 \mathrm{mg} \mathrm{kg}^{-1}$ and time intervals between ALA administration and the procedure of 2,6 or $16 \mathrm{~h}$ were used. The experiments were started at an i.p. ALA dose of $200 \mathrm{mg} \mathrm{kg}^{-1}$ and a time of $2 \mathrm{~h}$. These conditions were expected to induce evident macroscopic and microscopic damage to intra-abdominal organs $[7,19,20]$, thus giving an insight into the kind of tissue/cellular damage to be expected. The dose of i.p. ALA was subsequently decreased $(100,25,20,15,10,5,1)$ until no more signs of macroscopic and microscopic damage to healthy tissue were found. This dose was considered to be the maximal tolerable dose (MTD) of i.p. ALA for a $2 \mathrm{~h}$ interval between administration and the surgical procedure. Based on the information gained from the $2 \mathrm{~h}$ interval series, experiments at the other time intervals $(6,16$ and $24 \mathrm{~h})$ were performed in the opposite way by starting at a low, probably non-toxic, dose of ALA, and increasing this dose until signs of macroscopic and/or microscopic damage were found and the MTD could be established. The MTD estimated from the i.p. experiments was used as the starting dose for the i.v. dose-finding experiments. Two rats were used at each dose level, and the MTD for each time interval was confirmed by an additional two rats. Control groups were also included, which received no ALA but otherwise received the same treatment.

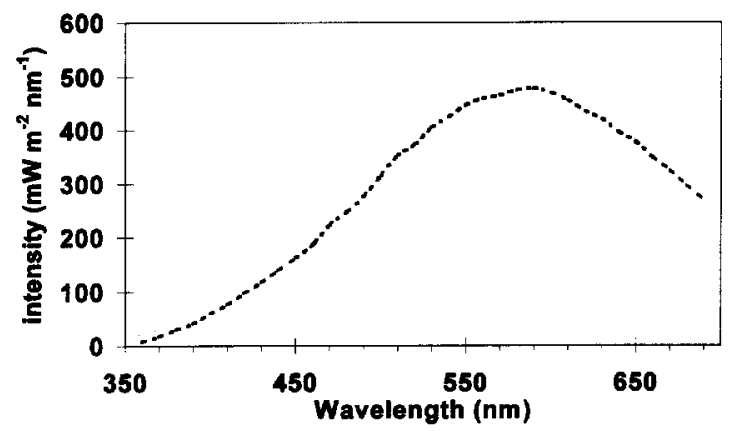

Fig. 1. Power spectrum of the Hanau operating-room lamp used. Total output $100 \mathrm{~W} \mathrm{~m}^{-2}$ in focal plane.

All experiments were performed under the same illumination conditions using a standard o.r. light source (Original Hanau, model 3120). The power spectrum of this lamp, depicted in Fig. 1, was measured with a calibrated optical multichannel analyzer (Oriel, model 77702, spectrograph MS125, calibrated with a model 63355 calibration lamp). We calculated the effective power spectrum, defined as the product of the (normalized) excitation spectrum of the photosensitizer and the power spectrum of the light source [22]. The effective power spectrum expresses the effectiveness of a certain light source for the excitation of a certain photosensitizer. Using this expression, extrapolation of our results to other clinical settings is possible. For future purposes we also calculated the effective power spectrum of an endoscope light source.

\subsection{Experiments}

Prior to the surgical procedure, the rats that received i.v. ALA were anesthesized with Ketamine $\left(90 \mathrm{mg} \mathrm{kg}^{-1}\right.$ body weight, i.m.), Xylazine ( $10 \mathrm{mg} \mathrm{kg}^{-1}$ body weight, i.m.) and Atropine $\left(0.05 \mathrm{mg} \mathrm{kg}^{-1}\right.$ body weight, i.m.). The rats that received i.p. ALA were anesthesized by Dormicum ( $1 \mathrm{mg}$ $\mathrm{kg}^{-1}$ body weight i.p.) and Hypnorm (1 $\mathrm{mg} \mathrm{kg}^{-1}$ body weight i.p.). A midline incision was made from xiphoid to symphysis. A surgical retractor was placed in situ, which kept the internal abdominal wall folded outside and the intraabdominal organs exposed to the light source. This situation was maintained for $2 \mathrm{~h}$ with the light source at a working distance of $90 \mathrm{~cm}$. Two animals were treated simultaneously. The intra-abdominal organs were kept moist during the procedure with Ringers solution at $37^{\circ} \mathrm{C}$. To maintain body temperature, an electrical temperature-controlled underlay $\left(37^{\circ} \mathrm{C}\right)$ was used. During the procedure, liver, small and large intestines, peritoneum, skin and bladder were macroscopically observed for changes and signs of damage according to a standard list. After $2 \mathrm{~h}$ the surgical procedure was ended and the abdominal skin was sutured. The rats were housed in dimmed lighting. Post-operative pain was suppressed with Buprenorphin $\left(0.2 \mathrm{ml} \mathrm{kg}{ }^{-1}\right.$ i.m). The rats were sacrificed after $72 \mathrm{~h}$. At re-laparotomy the above-mentioned organs were observed for macroscopic changes. Samples were taken from the liver, small and large intestines, peritoneum, skin 
and bladder in a standardized way and stored in formalin before being sectioned ( $5 \mu \mathrm{m}$ sections) and stained (hematoxylin and eosin) for histological examination.

\section{Results}

\subsection{General observations}

In the period between administration of ALA and the start of surgery, the rats showed no sign of discomfort, although the ALA solution was acidic ( $\mathrm{pH} 2.5-5$ ). In one rat, a small amount of ALA solution was accidentally injected into subcutis and muscle. Necrosis of the muscle was only seen at 16 $\mathrm{h}$ after administration of ALA. I.v. injection in the tail vein sometimes resulted in a local hemorrhage.

\subsection{Macroscopic observations during the procedure}

In the control group, which received no ALA and/or saline solution, no changes to intra-abdominal organs could be observed during and after $2 \mathrm{~h}$ of illumination.

Two animals ( $200 \mathrm{mg} \mathrm{kg}^{-1}$ i.p., $2 \mathrm{~h}$ interval) died almost immediately after the procedure. The macroscopic observations during the illumination procedure are summarized in Fig. 2 for the various combinations of ALA dose $\left(\mathrm{mg} \mathrm{kg}^{-1}\right)$ and time interval (hours) between administration and illumination.

In the exposed (illuminated) part of the liver, we observed a change in color during illumination, either reddening or blanching and edema, after administration of $\geq 5 \mathrm{mg} \mathrm{kg}^{-1}$ for a time interval of $2 \mathrm{~h}, \geq 20 \mathrm{mg} \mathrm{kg}^{-1}$ for a time interval of $6 \mathrm{~h}$ and $\geq 200 \mathrm{mg} \mathrm{kg}^{-1}$ for a time interval of $16 \mathrm{~h}$. In all cases, the surface remained intact. A change in appearance of the small and large intestines was observed after administration of $\geq 20 \mathrm{mg} / \mathrm{kg}$ ALA for intervals of 2 and $6 \mathrm{~h}$ and $\geq 200 \mathrm{mg} \mathrm{kg}^{-1}$ at $16 \mathrm{~h}$. A swelling of the intestinal wall was typically seen, together with focalized hemorrhages on the outer surface. The visual appearance of the other intraabdominal organs and the abdominal wall including the skin was not macroscopically changed during the procedure.

The macroscopic observations during illumination after i.v. administration of ALA were comparable with those obtained in the i.p. group (see Fig. 3).

\subsection{Macroscopic observations after $72 h$}

In the control group, which received no ALA and/or saline solution, no changes to intra-abdominal organs were observed. During the three days after the procedure, the animals showed no change in behavior and weight loss was limited to a maximum of $15 \%$, which was not different from the weight loss seen after surgery alone.

The macroscopic observations after $72 \mathrm{~h}$ are summarized in Figs. 4 and 5. Findings were comparable with those found during the surgical procedure. The discoloration of the liver

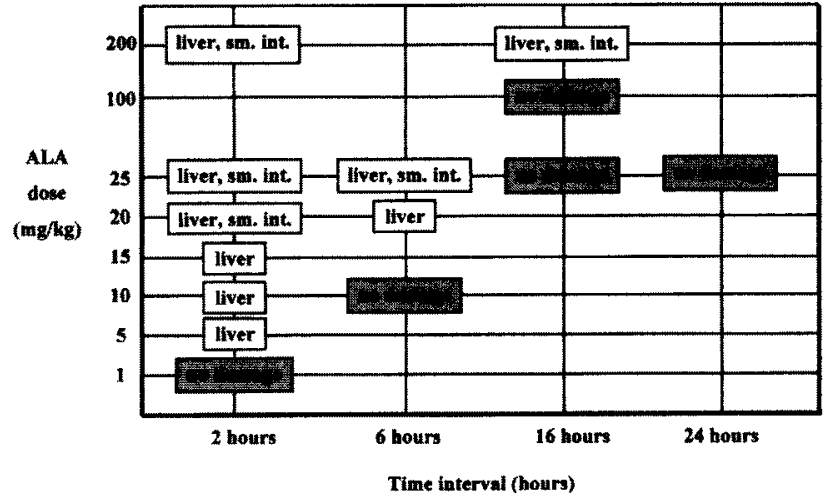

Fig. 2. The combinations of ALA doses ( $\mathrm{mg} \mathrm{kg}^{-1}$ ) and time intervals (hours) between i.p. administration and treatment at which damage is observed (macroscopic damage, during illumination). The organs that were judged to be damaged are named in the boxes.

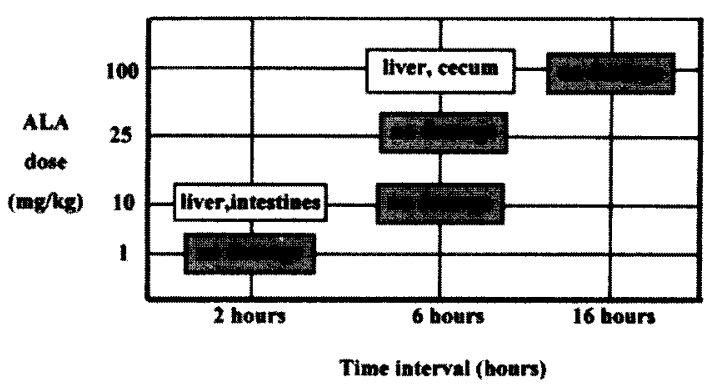

Fig. 3. The combinations of ALA doses $\left(\mathrm{mg} \mathrm{kg}^{-1}\right)$ and time intervals (hours) between i.v. administration and treatment at which damage is observed (macroscopic damage, during illumination). The organs that were judged to be damaged are named in the boxes.

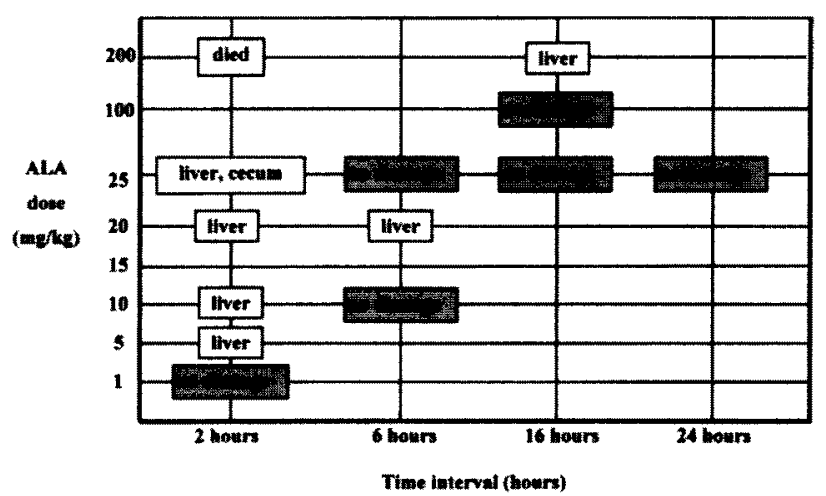

Fig. 4. The combinations of ALA doses $\left(\mathrm{mg} \mathrm{kg}^{-1}\right)$ and time intervals (hours) between i.p. administration and treatment at which damage is observed (macroscopic damage, $72 \mathrm{~h}$ after illumination). The organs that were judged to be damaged are named in the boxes.

observed during the illumination was in most cases still noticeable after $72 \mathrm{~h}$. Additionally, in these cases a white line was observed, which delineated the illuminated area. The swelling of the intestines had disappeared in all cases. In a few cases a faint reddish discoloration was still present. No macroscopic changes to the other intra-abdominal organs could be observed. Findings were similar for both i.v.- and i.p.-treated rats. 


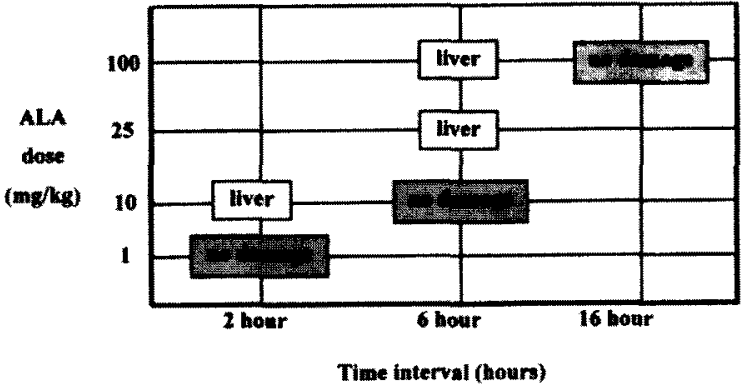

Fig. 5. The combinations of ALA doses $\left(\mathrm{mg} \mathrm{kg}^{-1}\right)$ and time intervals (hours) between i.v. administration and treatment at which damage is observed (macroscopic damage, $72 \mathrm{~h}$ after illumination). The organs that were judged to be damaged are named in the boxes.

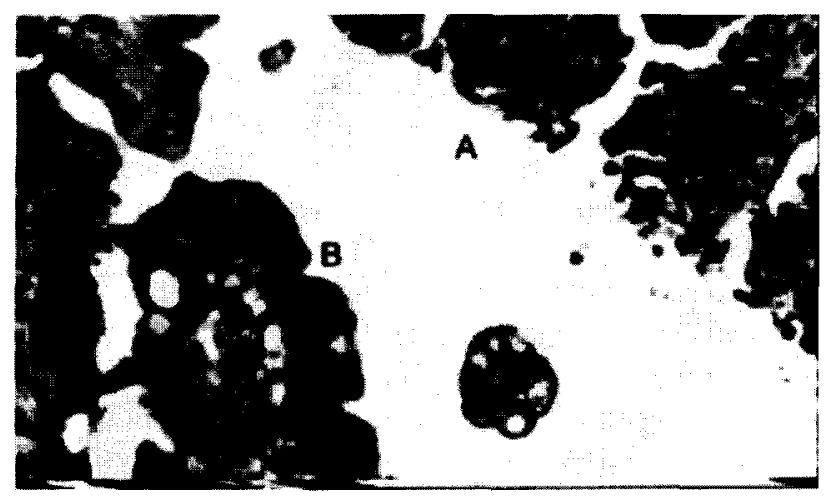

Fig. 6. Small-intestine wall (dose i.p. $25 \mathrm{mg} \mathrm{kg}^{-1}$, interval $2 \mathrm{~h}$ ). A: on the illuminated side the mucosa has disappeared, while at the dark side (B, shielded from the light by feces) the mucosa is still intact. The other layers remain intact.

\subsection{Microscopic observations}

Microscopic damage was limited to the liver and the intestine (wall). A typical example of the changes due to whitelight illumination of the small intestine is shown in Fig. 6 (i.p., $25 \mathrm{mg} \mathrm{kg}^{-1}, 2 \mathrm{~h}$ interval). The outer layers, muscularis and submucosa, were intact, while the mucosa on the side of the bowel exposed to the light source (A) has completely disappeared. The opposite mucosa, shielded by feces from the light source, remained intact. Fig. 7 shows the damage to the liver of the same rat. A clearly demarcated area with cell damage is seen at the illuminated side. The thickness of this damaged layer is approximately $0.4 \mathrm{~mm}$. The damage to both the liver and intestines was focal. In one rat (i.v., $25 \mathrm{mg} \mathrm{kg}^{-1}$,
$6 \mathrm{~h}$ interval) a necrotic superficial vessel wall was observed in the liver. We have not seen any abnormalities in other abdominal organs. In the control group no damage was seen to illuminated organs.

\subsection{Maximum tolerable dose (MTD)}

To obtain the MTD (no sign of macroscopic or microscopic damage) for the tandem dose of ALA and time interval, we combined the results from Figs. 2 and 3 (macroscopic observations during the procedure), Figs. 4 and 5 (macroscopic observations after $72 \mathrm{~h}$ ) and the microscopic observations. For the $2 \mathrm{~h}$ interval the MTD was established at 1 $\mathrm{mg} \mathrm{kg}{ }^{-1}$; for the $6 \mathrm{~h}$ interval the MTD was established at 10 $\mathrm{mg} \mathrm{kg}{ }^{-1}$ and for the $16 \mathrm{~h}$ interval the MTD was established at $100 \mathrm{mg} \mathrm{kg}^{-1}$.

\subsection{Effective power spectrum}

The effective power spectrum of the o.r. light used in these studies and the relative excitation spectrum of PpIX are depicted in Fig. 8. For comparison, Fig. 9 shows the effective power spectrum of an endoscope white-light source ( $100 \mathrm{~W}$ xenon) with the absolute power spectrum measured $5 \mathrm{~cm}$ from the distal end.

\section{Discussion}

Photodetection using ALA-induced PpIX may be an attractive new diagnostic development in ovarian cancer diagnostic strategies. Photodetection can be added to current staging procedures like laparoscopy or laparotomy, enabling the localization of macroscopically non-visible metastases. However, fluorescent diagnostics carry a risk of damage to tissue due to the photosensitization properties of PpIX. This risk is determined by the illumination properties (power and wavelength) of the light source, the dose of photosensitizer, the interval before illumination and the type of tissue/organ. During the photodetection-assisted staging procedures, which can last several hours, the abdominal cavity will be exposed not only to blue light from the excitation source but also to intense white light from the endoscopic light sources or o.r. lamps. In order to minimize the risk of phototoxic

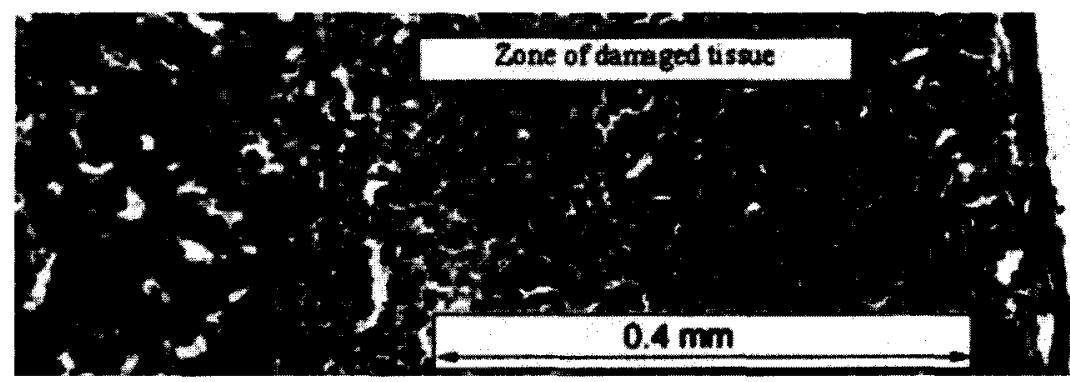

Fig. 7. Zone of cell damage at the illuminated side of the liver (dose i.p. $25 \mathrm{mg} \mathrm{kg}^{-1}$, interval $2 \mathrm{~h}$ ). 


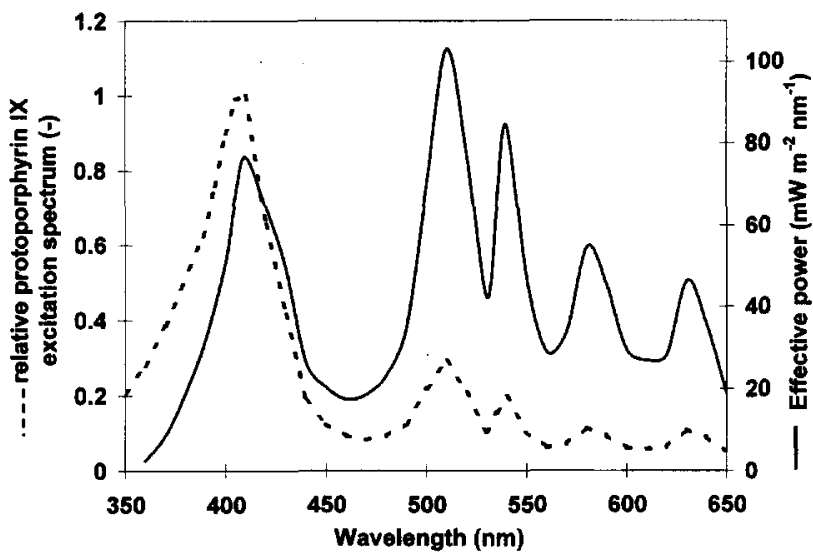

Fig. 8. The relative absorption spectrum of protoporphyrin IX (dashed line) that we used to calculate the effective power spectrum of the operating-room lamp (full line).

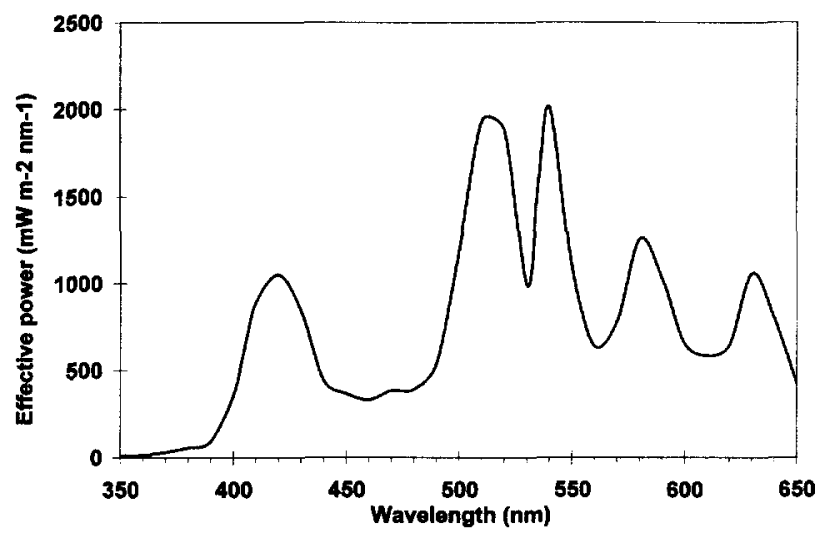

Fig. 9. The effective power spectrum of a white-light endoscope (xenon).

damage in future photodetection-assisted staging procedures, we have established safe ALA dose/time intervals when using white-light sources in a rat model.

Safe doses (MTDs) were established at 1,10 and $100 \mathrm{mg}$ $\mathrm{kg}^{-1}$ for intervals of 2,6 and $16 \mathrm{~h}$, respectively, for both i.v. and i.p. routes of administration. As can be expected, the shorter the time interval, the lower the MTD for ALA. Low dosages of ALA may not be very useful in photodetection. The success of photodetection diagnostics is based on differences in concentrations of photosensitizer between normal and tumor tissue and low ALA doses may not give enough contrast. In that case, a higher ALA dose at a longer time interval should be used for clinical applications. Recently Hornung published on photodetection diagnostics of carcinomatosis in the (rat) abdominal cavity using ALA in combination with a blue excitation light source [1]. He observed the best contrast between tumor fluorescence and normal (peritoneum) fluorescence in the abdominal cavity $3 \mathrm{~h}$ after administration of $100 \mathrm{mg} \mathrm{kg}^{-1}$ ALA. The combination of this relatively high drug dose and short time interval (between administration of ALA and illumination) would result in considerable tissue damage on exposure to the illumination conditions that we used in our experiments.
When evaluating the safety of photodetection diagnostics, both the severity and the (ir) reversibility of any induced damage of healthy tissue have to be taken into account. In this study we considered any macroscopic or microscopic change in organs as phototoxic damage. Phototoxic damage appeared to be restricted to the liver and small bowel. The observed damage would probably have little impact on the functionality of the organs involved, particularly in the liver, where damage was limited to a superficial layer $(0.4 \mathrm{~mm})$. Due to the structure and size of the liver, this would have few functional consequences. Damage to the intestinal wall was limited to the mucosa, whereas the muscular wall remained intact. Our study was limited to an evaluation of damage at $72 \mathrm{~h}$ after illumination. Longer follow-up would, presumably, indicate regeneration of the damaged mucosa from unaffected areas. The chance of severe complications, like bowel perforation, therefore seems to be limited. Nevertheless, our results in rats indicate that caution should be taken when applying photodetection diagnostics using intense light sources. So far no clinical studies mention any phototoxic damage as a possible side effect of photodetection diagnostic procedures, even if white-light sources are used. The discrepancy between our rat data and these clinical observations could be related to the larger mass of human organs, with any damage being restricted to superficial layers without functional consequences. However, this has not been systematically evaluated and the impression remains that possible damage might be overlooked in clinical studies. Our intention was not to give absolute safe doses for humans, but rather to demonstrate the potential for toxic side effects during photodetection procedures with high doses (and short intervals) of ALA. The study also indicates conservative safe doses where no damage is to be expected.

\section{Suggestions of precautions}

To avoid phototoxicity in normal tissues during photodetection procedures, the following should be considered: phototoxicity is determined by the light-source properties, the dose of ALA and the interval between application and surgical procedure $[22,23]$. In photodetection diagnostics via laparoscopy and laparotomy the use of white light cannot be avoided. Modification of the light source by using filters for blocking the blue part of the light source spectrum is not very effective, as only a small influence on the effective power is obtained. In contrast to a common misunderstanding, the white-light source of an endoscope is about 20 times more effective in causing damage to sensitized tissue than an o.r. lamp (as demonstrated in Fig. 9). This leaves the time of exposure and the drug dose as potential variables in diminishing damage. In a clinical setting a time interval has to be used where the ALA dose is below the MTD but sufficient to enable discrimination between normal and tumor tissue. 


\section{Acknowledgements}

This work was supported in part by the Dutch Cancer Foundation (AVL, klinische startgelden, 1995) and in part by the European Commission, Biomed 2, project 96-2260.

\section{References}

[1] R. Hornung, A.L. Mayor, M. McHale, L.H.L. Liaw, L.A. Sabiniano, B.J. Tromberg, M.W. Berns, Y. Tadir, In vivo detection of metastatic ovarian cancer by means of 5-aminolevulinic acid-induced fluorescence in a rat model, J. Am. Assoc. Gyn. Lapar. 5 (2) (1998) 141148.

[2] D.L. Campbell, E.F. Gudgin-Dickson, P.G. Forkert, R.H. Pottier, J.C. Kennedy, Detection of early stages of carcinogenesis in adenomas of murine lung by 5 -aminolevulinic acid-induced protoporphyrin IX fluorescence, Photochem. Photobiol. 64 (1996) 676-682.

[3] A. Leunig, K. Rick, H. Stepp, R. Gutmann, G. Alwin, R. Baumgartner J. Feyh, Fluorescence imaging and spectroscopy of 5-aminolevulinic acid induced protoporphyrin IX for the detection of neoplastic lesions in the oral cavity, Am. J. Surg. 172 (1996) 674-677.

[4] M. Kriegmair, R. Baumgartner, R. Knüchel, P. Steinbach, A. Eshan, W. Lumper, F. Hofstäter, A. Hofstetter, Fluorescence photodetection of neoplastic urothelial lesions following intravesical instillation of 5 aminolevulinic acid, Urology 44 (1994) 836-841.

[5] M. Kriegmair, R. Baumgartner, R. Knüchel, H. Stepp, F. Hofstädter, A. Hofstetter, Detection of early bladder cancer by 5 -aminolevulinic acid induced porphyrin fluorescence, J. Urol. 155 (1996) 105-110.

[6] D. Kessel, Porphyrin localization: a new modality for detection and therapy of tumors, Biochem. Pharmacol. 33 (1984) 1389-1393.

[7] J. Bedwell, A.J. MacRobert, D. Phillips, S.G. Bown, Fluorescence distribution and photodynamic effect of ALA-induced PPIX in the DHM rat colonic tumour model, Br. J. Cancer 65 (1992) 818-824.

[8] F. Cairnduff, D.J.H. Roberts, B. Dixon, S.B. Brown, Response of a rodent fibrosarcoma to photodynamic therapy using 5-aminolevulinic acid or polyhaematoporphyrin, Int. J. Radiat. Biol. 7 (1995) 93-99.

[9] W.E. Grant, C. Hopper, A.J. MacRobert, P.M. Speight, S.G. Bown, Photodynamic therapy of oral cancer: photosensitization with systemic aminolevulinic acid, Lancet 342 (1994) 147-148.

[10] J. Leveckis, J.L. Burn, N.J. Brown, M.W.R. Reed, Kinetics of endogenous protoporphyrin IX induction by aminolevulinic acid: preliminary studies in the bladder, J. Urol. 152 (1994) 550-553.

[11] B.W. Henderson, L. Vaughan, D.A. Bellnier, H.L.L.M. van Leengoed, P.G. Johnson, A.R. Oseroff, Photosensitization of murine tumor, vas- culature and skin by 5-aminolevulinic acid-induced porphyrin, Photochem. Photobiol. 62 (1995) 780-789.

[12] H.J. van Staveren, J.F. Beek, C.W.J. Verlaan, A. Edixhoven, A.E. Saarnak, H.J.C.M. Sterenborg, T.M. de Reijke, G.B. de la Riviere, S.L. Thomsen, M.J.C. van Gemert, W.M. Star, Whole bladder wall photodynamic therapy using 5-ALA: an experimental study in pigs, SPIE Proc. 2625 (1996) 70-76.

[13] K. König, H. Meyer, H. Schneckenburger, A. Rück, The study of endogenous porphyrins in human skin and their potential for photodynamic therapy by laser induced fluorescence spectroscopy, Lasers Med. Sci. 8 (1993) 127-132.

[14] J.C. Kennedy, R.H. Pottier, D.C. Pross, Photodynamic therapy with endogenous protoporphyrin IX: basic principles and present clinical experience, J. Photochem. Photobiol. B: Biol. 8 (1990) 143-148.

[15] N. van der Veen, H.L.L.M. van Leengoed, W.M. Star, In vivo fluorescence kinetics and photodynamic therapy using 5 -aminolevulinic acid-induced porphyrin: increased damage after multiple irradiations, Br. J. Cancer 70 (1996) 867-872.

[16] Q. Peng, T. Warloe, J. Moan, H. Heyerdahl, H.B. Steen, J.M. Nesland, K.E. Giercksky, Distribution of 5-aminolevuline acid-induced porphyrins in noduloulcerative basal cell carcinoma, Photochem. Photobiol. 62 (1995) 906-913.

[17] J. Regula, A.J. MacRobert, A. Gorchein, G.A. Buonaccorsie, S.M. Thorpe, G.M. Spencer, A.R.W. Hatfield, S.G. Bown, Photosensitisation and photodynamic therapy of oesophaegeal, duodenal and colorectal tumours using 5-aminolevulinic acid induced porphyrin IX a pilot study, Gut 36 (1995) 67-75.

[18] R.A. Steiner, Y. Tadir, B.J. Tromberg, T. Krasieva, A.T. Ghazains, P Wyss, M.W. Berns, Photosensitization of the rat endometrium following 5-aminolevulinic acid induced photodynamic therapy, Lasers Surg. Med. 18 (1996) 301-308.

[19] C.S. Loh, J. Bedwell, A.J. MacRobert, N. Krasner, D. Phillips, S.G. Bown, Photodynamic therapy of the normal rat stomach: a comparative study between di-sulphonated aluminium phthalocyanine and 5aminolevulinic acid, Br. J. Cancer 66 (1992) 452-462.

[20] C.S. Loh, A.J. MacRobert, J. Bedwell, J. Regula, N. Krasner, S.G Bown, Oral versus intravenous administration of 5-aminolevulinic acid for photodynamic therapy, Br. J. Cancer 68 (1993) 41-51.

[21] B.A. Goff, R. Bachor, N. Kollias, T. Hasan, Effects of photodynamic therapy with topical application of 5-aminolevulinic acid on normal skin of hairless guinea pigs, J. Photochem. Photobiol. B: Biol. 15 (1992) 239-251.

[22] H. Moseley, Total effective fluence: a useful concept in photodynamic therapy, Lasers Med. Sci. 11 (1996) 143-193.

[23] L.I. Grossweiner, Light dosimetry model for photodynamic therapy treatment planning, Lasers Surg. Med. 11 (1991) 165-173. 